\title{
A phase I study of bortezomib and temozolomide in patients with advanced solid tumors
}

\author{
J. Portnow, P. Frankel, S. Koehler, P. Twardowski, S. Shibata, C. Martel, R. Morgan, M. \\ Cristea, W. Chow, D. Lim, V. Chung, K. Reckamp, L. Leong, and T. W. Synold \\ Department of Medical Oncology, City of Hope, 1500 East Duarte Road, Duarte, CA 91030, USA \\ J. Portnow: jportnow@coh.org
}

\begin{abstract}
Purpose-The primary objective was to determine the maximum tolerated doses (MTDs) of the combination of bortezomib and temozolomide in patients with solid tumors. The secondary objective was to evaluate the pharmacokinetics (PK) of bortezomib with and without concurrent hepatic enzyme-inducing anticonvulsants (HEIAs).
\end{abstract}

Methods-Bortezomib was administered on days 2, 5, 9, and 12; temozolomide on days 1-5 of a 28 -day cycle. Dose escalation proceeded using a standard $3+3$ design. Patients with primary or metastatic brain tumors were eligible and were stratified based on whether they were taking HEIAs or not.

Results-Of the 25 patients enrolled, 22 were not taking HEIAs. MTDs were only given to patients not receiving HEIAs. Dose-limiting toxicities (DLTs) consisted of grade-3 constipation, hyponatremia, fatigue, elevated hepatic enzymes, and grade- 4 neutropenia, thrombocytopenia, constipation, and abdominal pain. Stable disease ( $>8$ weeks) was observed in 5 patients. Bortezomib systemic clearance $\left(\mathrm{CL}_{\text {sys }}\right)$ on day 9 was $51 \%$ of the $\mathrm{CL}_{\text {sys }}$ on day $2(P<0.01)$ Similarly, the normalized area under the concentration-time curve (norm AUC) on day 9 was 1.9 times the norm AUC on day $2(P<0.01)$. The median bortezomib $\mathrm{CL}_{\text {sys }}$ on days 2 and 9 was significantly higher $(P<0.04)$ in patients taking HEIAs, and the median norm AUC was correspondingly lower $(P<0.04)$.

Conclusions-The MTDs for the combination of bortezomib and temozolomide in patients not taking HEIAs are 1.3 and $200 \mathrm{mg} / \mathrm{m}^{2}$, respectively. The rate of bortezomib elimination in patients taking HEIAs was increased twofold. Additional trials are needed to better define the optimal dosing in such patients.

\section{Keywords}

Bortezomib; Temozolomide; Phase I; Pharmacokinetics; Hepatic enzyme-inducing anticonvulsants

\section{Introduction}

Proteasomes are present in the nucleus and cytoplasm of all eukaryotic cells [1] and play an essential role in the processing of cellular proteins. Included among the numerous proteins that the proteasome degrades are ones involved in progression of the cell cycle and

\footnotetext{
(C) Springer-Verlag 2011

Correspondence to: J. Portnow, jportnow@coh.org.

Conflicts of interest No financial conflict of interest exists for any of the authors.
} 
protection of the cell from apoptosis [2]. By interfering with the orderly degradation of these regulatory proteins, proteasome inhibitors can arrest tumor growth and metastases [3].

The proteasome inhibitor bortezomib (Velcade; formerly PS-341, LDP-341, MLN341) is a dipeptide boronic acid analogue that has received approval from the Food and Drug Administration for treatment of recurrent multiple myeloma (2003) and mantle cell lymphoma (2006). Pre-clinical studies have shown that bortezomib also has activity against a wide array of solid tumors including prostate [4, 5], pancreatic [5-9], lung [10, 11], head and neck [12, 13], colon [14], breast [15, 16], bladder [17], and ovarian [4, 18] cancers. In addition to single agent activity against solid tumors, in vitro studies of bortezomib in combination with cytotoxic chemotherapy agents such as irinotecan $[6,14]$ and gemcitabine [7] have demonstrated efficacy, and multiple subsequent phase I studies [19-24] in patients with solid tumors have been conducted.

Temozolomide is an atypical alkylating agent most commonly used for the treatment of gliomas [25] and advanced melanoma [26]. Temozolomide causes the formation of methyl adducts resulting in futile mismatch repair cycles that initiate the process of apoptosis [27]. Theoretically, the mechanisms of action of temozolomide and bortezomib may be synergistic, since temozolomide induces apoptosis and bortezomib blocks a cancer cell's survival response to the initiation of apoptosis. There are preclinical data demonstrating efficacy of this combination in vitro and against human melanoma xenografts [28].

This phase I study of bortezomib and temozolomide was conducted in patients with refractory solid tumors to determine the maximum tolerated doses (MTDs) and doselimiting toxicities (DLTs) of this combination. A secondary objective of this study was to determine whether hepatic enzyme-inducing anticonvulsants (HEIAs) affect the metabolism of bortezomib.

\section{Patients and methods}

\section{Patient selection}

Eligibility requirements for this phase I study included patients having to be at least 18 years of age with a metastatic or unresectable solid tumor for which standard curative or palliative measures were no longer effective. Patients with primary or metastatic brain tumors, recurring after radiation therapy, were eligible to participate. There was no limit to the number of prior therapies, and either measurable or non-measurable disease was allowed. Brain tumor patients who required the use of anti-seizure medications or corticosteroids to control cerebral edema had to be on stable doses of these medications for at least 1 week prior to enrollment. A minimum of 4 weeks had to elapse between receiving a previous chemotherapy (6 weeks from a nitrosourea-containing chemotherapy), immunotherapy, or radiation and enrollment. Other conditions required for study participation included (1)

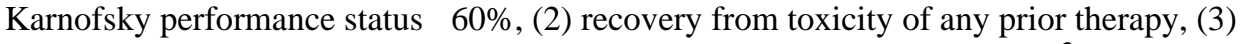
adequate bone marrow function (absolute neutrophil count $\geq 1,500$ cells $/ \mathrm{mm}^{3}$, platelet count $\geq 100,000$ cells $/ \mathrm{mm}^{3}$ ), (4) adequate hepatic function (total bilirubin $\preceq 2.0 \mathrm{mg} / \mathrm{dl}$, serum levels of aspartate aminotransferase $\leq 4 \times$ the institutional upper limit of normal), and (5) adequate renal function (serum creatinine $\leq .5 \mathrm{mg} / \mathrm{dl}$ ).

Patients were excluded from participating in this study if they (1) were receiving chemotherapy, radiation therapy, or were enrolled in another clinical trial; (2) were pregnant or breast-feeding; (3) were receiving anti-retroviral therapy for the treatment of human immunodeficiency virus; (4) had a history of sensitivity to boron or mannitol; or (5) had a serious medical or psychiatric illness that could, in the investigator's opinion, potentially interfere with the completion of treatment according to the protocol. In accordance with the 
Declarations of Helsinki, the clinical protocol and informed consent document were approved by the City of Hope Institutional Review Board. All patients gave written informed consent prior to study participation. This study was registered with http:// www.clinicaltrials.gov, identifier NCT00544284.

\section{Treatment plan}

This was a single center, phase I dose-finding study of bortezomib in combination with temozolomide. A treatment cycle was defined as 28 days in length. Bortezomib (provided by Millennium Pharmaceuticals, Cambridge, MA) was administered by rapid intravenous push on days 2, 5, 9, and 12. Temozolomide (provided by Merck \& Co., Inc. [formerly ScheringPlough Corp.]) was taken orally by study patients at bedtime on days $1-5$ of each cycle. All patients premedicated themselves with ondansetron, $8 \mathrm{mg}$ orally, $30 \mathrm{~min}$ prior to each dose of temozolomide for nausea prophylaxis.

Different dosing schedules of temozolomide are used in the clinical setting. For example, the intermittent dosing schedule of temozolomide, daily for 5 consecutive days, every 28 days, is used as part of the standard treatment for patients with newly diagnosed glioblastoma after completing radiation therapy [25]. It is also the FDA-approved dosing schedule for the treatment of recurrent anaplastic astrocytoma and metastatic melanoma, and it has been evaluated in patients with recurrent brain metastases as well $[29,30]$. There is also a continuous dosing schedule of temozolomide, using a lower dose daily for up to 49 days, which is typically administered concurrently with brain radiation for treatment of newly diagnosed glioblastoma, and it has also been studied in combination with radiation in patients with brain metastases [31]. Since radiation was not a component of the treatment being assessed in this clinical trial, we chose to combine bortezomib with the standard intermittent dosing schedule of temozolomide.

Five dose levels were tested (see Table 2 for the dose escalation schedule). Because of the potential overlapping toxicity of bone marrow suppression, dose escalation of the combination began below the respective individual MTDs of $1.6 \mathrm{mg} / \mathrm{m}^{2}$ for bortezomib [32, $33]$ and $200 \mathrm{mg} / \mathrm{m}^{2}$ for temozolomide [34, 35]. The dose of temozolomide was increased from $100 \mathrm{mg} / \mathrm{m}^{2}$ to the standard recommended dose for the intermittent schedule of temozolomide, $200 \mathrm{mg} / \mathrm{m}^{2}$, by $50 \mathrm{mg} / \mathrm{m}^{2}$ increments through the first 3 planned dose levels, while the dose of bortezomib remained at $1.0 \mathrm{mg} / \mathrm{m}^{2}$. After the established standard dose for temozolomide $\left(200 \mathrm{mg} / \mathrm{m}^{2}\right)$ was reached, bortezomib was escalated in dose levels 4 and 5 . For this phase I study, patients were stratified into 2 groups based on whether they were taking HEIAs (phenytoin, carbamazepine, phenobarbital, primidone or oxcarbazepine) concomitantly (group A) or not (group B).

\section{Definition of DLT and rules for dose escalation}

DLT was defined as any grade- 3 or grade- 4 non-hematologic toxicity or any grade- 4 hematologic toxicity possibly related to the study drugs, excluding anemia, although its occurrence was documented along with other toxicities. DLT was based on the first course of treatment. Toxicity was graded according to the NCI Common Terminology Criteria for Adverse Events version 3.0. To be evaluable for toxicity, a patient must have received at least 1 dose of chemotherapy during cycle 1 .

A minimum of 3 patients were to be treated at each dose level. If a patient did not receive all of the planned chemotherapy for cycle 1, due to either non-compliance or toxicity, s/he would be replaced for purposes of dose escalation. Patients who did not experience any DLT had to be observed for a minimum of 4 weeks from the start of the first cycle before moving to the next dose level. If DLT attributable to the study drugs was experienced in exactly 1 of 
3 patients, then 3 more patients (for a total of 6) would be treated at that dose level. If no additional DLT was observed at the expanded dose level (i.e., 1 out of 6 with DLT), then the dose would be escalated. Dose escalation would terminate as soon as 2 or more patients experienced any DLT attributable to the study drugs, at a given dose level. The study would finish when 6 patients were treated at the next lower dose level, and at most 1 patient out of those 6 patients experienced a DLT.

The same dose escalation schema was used for groups A and B; however, the drugs were dose-escalated separately in the 2 groups. We hypothesized that if HEIAs induce the metabolism of bortezomib, leading to enhanced clearance of bortezomib, then serum drug levels would be lower, resulting in less toxicity occurring at a given dose level compared to patients not taking HEIAs. Therefore, study rules for dose escalation specified that if group $\mathrm{B}$ was at a higher dose level, then group A could be advanced to the same dose level. Therefore, if no DLTs were observed in group A, and group B was testing a higher dose or if the MTD for group B had been determined, and it was higher than the current level for group A, then group A would be escalated to the testing dose (or the MTD if applicable) for group B with the next patient to accrue to group A.

A DLT in group A (taking HEIAs) would count at least as a DLT in group B for dose deescalation purposes, as patients in group A should not be exposed to more drug than group B due to the HEIAs, but not vice versa. While a DLT in group B would not be viewed as a simultaneous DLT for group A, a DLT in group B could be used to reduce the dose in group A in order to explore a lower dose before deciding to continue escalation at the higher dose. Such decision would be made at the discretion of the principal investigator to help protect patient safety.

\section{Statistical considerations}

All statistical tests used the Wilcoxon rank sum test (2-sided) unless otherwise specified. Statistical calculations were carried out using S-Plus 7.0.

\section{Patient assessments}

Symptom assessments and physical examinations were performed prior to each treatment cycle. During the first cycle, complete blood counts were monitored on days 2, 5, 9, 12, 16, and 23. Results of hematologic parameters on day 9 were used to make any dose adjustments of bortezomib on days 9 and 12. Radiographic assessments were performed prior to starting study treatment and then every 8 weeks. To be evaluable for response, patients were required to complete at least 2 cycles of study treatment. Tumor responses were determined using the RECIST criteria [36] for measurable disease outside of the brain and the Macdonald criteria [37] for assessing disease within the brain.

\section{Sample collection}

On days 2 and 9 of cycle 1, blood samples were drawn immediately before the bolus infusion of bortezomib and then 5, 10, 15, $30 \mathrm{~min}$, and 1, 2, 4, 6, 8, and $24 \mathrm{~h}$ after dosing. Additional blood samples were drawn before administration of bortezomib on days 5 and 12. At each time point, $5 \mathrm{ml}$ of peripheral blood was collected in $\mathrm{K}_{3}$ EDTA tubes and mixed by gently inverting $4-6$ times. The anticoagulated blood was centrifuged to separate plasma. Equal volumes of the plasma were transferred to appropriately labeled cryovials and frozen immediately at $-70^{\circ} \mathrm{C}$. Plasma samples were shipped to Millennium Pharmaceuticals for analysis of bortezomib concentrations. To confirm that patients in group A were taking their anticonvulsants and that these drug concentrations were within therapeutic range, anticonvulsant levels were drawn at the start of each cycle, and during cycle 1, trough levels of anticonvulsants were also drawn 10 min prior to the morning dose on days 2 and 9 . 


\section{Analytical method}

Bortezomib plasma concentrations were analyzed at Tandem Labs using a validated LCMS/MS assay [38]. Following addition of the internal standard ([13C9] bortezomib), plasma samples $(0.2 \mathrm{ml})$ were subject to liquid/liquid extraction with methyl tert-butyl ether and processed for LC-MS/MS analysis. Chromatography was performed using a Sunfire C8, 2.1 $\times 50 \mathrm{~mm}, 5 \mu \mathrm{m}$; Waters, Milford, MA) and a $0.1 \%$ formic acid/acetonitrile $/ 5 \% 25 \mathrm{mM}$ $\mathrm{NH}_{4} \mathrm{OAC}$ isopropanol mobile phase (flow rate $0.4 \mathrm{ml} / \mathrm{min}$ ), with elution of the analytes over a 30-40\% acetonitrile gradient. Mass spectrometric detection was performed with a mass spectrometer equipped with a turbo ion spray source (API 4000, MDS Sciex, Thornhill, Ontario), using multiple reaction monitoring in the positive ion mode $(\mathrm{m} / \mathrm{z}$ transitions of $367.1 \rightarrow 226.0$ for bortezomib and $376.1 \rightarrow 234.0$ for the internal standard). Calibration curves were linear from 0.1 to $25 \mathrm{ng} / \mathrm{ml}\left(r^{2} \geq 0.99\right)$. Samples with concentrations above the upper limit of quantitation were adequately diluted into the dynamic range of the assay with control matrix for bioanalysis. The interassay precision (CV) of the analytical quality control samples ranged from 8.0 to $10.3 \%$. The interassay accuracy, described as percent deviation, ranged from -1.0 to $2.0 \%$.

\section{Pharmacokinetic data analysis}

Pharmacokinetic (PK) parameters were determined from the measured plasma bortezomib concentration versus time data for each individual according to non-compartmental methods and using Microsoft Excel. The maximum plasma concentration $\left(\mathrm{C}_{\max }\right)$ was determined from the measured botezomib levels. The area under the concentration-time curve (AUC) was determined using the rule of linear trapezoids extrapolated to infinity using the elimination rate constant derived from the last four measured concentrations. The terminal half-life $\left(\mathrm{t}_{1 / 2}\right)$ was calculated from the elimination rate constant derived from the last four measured concentrations. Systemic clearance $\left(\mathrm{CL}_{\mathrm{sys}}\right)$ was calculated by dividing the total administered dose by the AUC extrapolated to infinity. To allow for comparisons across dose levels, $C_{\max }$ and AUC values were normalized to a dose of $1.3 \mathrm{mg} / \mathrm{m}^{2}$.

\section{Results}

\section{Patient characteristics}

Twenty-five patients were enrolled and received at least 1 dose of study treatment. Nine patients had CNS tumors: 4 with primary brain tumors ( 2 with high-grade gliomas, 1 with an ependymoma, and 1 with a meningioma) and 5 with brain metastases (primary sites of cancer: 3 lung, 1 breast, and 1 colon). Two patients in group B (1 at dose level 3 and 1 at dose level 4) did not complete the first cycle of treatment due to non-compliance and were replaced for the purpose of dose escalation but were included in the toxicity assessment. Table 1 lists the study patients' characteristics. Most were not taking HEIAs and so were enrolled in group B.

\section{Safety and tolerability}

The primary aim of this phase I study was to determine the MTDs for the combination of bortezomib and temozolomide in patients with solid tumors, including those involving the central nervous system (CNS). Table 2 summarizes the number of patients treated per dose level and the DLTs that occurred during the first cycle of treatment. As explained above, the same dose levels were used for groups A and B, with dose escalation proceeding independently for the 2 groups. However, if no DLTs had been observed in group A, and group B was testing a higher dose level, then group A could have accelerated escalation to the testing dose for group B. 
Patients enrolled in dose level 1 tolerated treatment well, experiencing only grade- 1 and grade- 2 toxicities. Four patients were enrolled in dose level 2 because 1 patient had to be replaced due to early death from disease progression. The first DLT was seen at dose level 3 in group B. This person had metastatic lung cancer involving his brain, bones, pancreas, and adrenal glands. He developed grade-4 abdominal pain and constipation attributed as possibly related to study treatment. Dose level 3 was then expanded to treat a total of 6 evaluable patients. One patient was replaced due to non-compliance with taking temozolomide.

No other DLTs occurred at that dose level, and the next 3 group B patients were enrolled in dose level 4. One of these patients was replaced due to non-compliance. No DLTs occurred among these first 4 patients, and so the next group B patient entered the study at dose level 5. This patient experienced DLTs consisting of grade- 3 constipation and hyponatremia.

The plan was then to expand dose level 5 to treat 6 patients from group B. However, the next patient to enroll in the study was in group A and was treated at dose level 5. This patient also developed DLTs consisting of grade-4 neutropenia and thrombocytopenia as well as grade-3 fatigue. As per the protocol's rules for dose escalation, a DLT in group A is counted as a DLT in group B as well; therefore, group B was de-escalated to dose level 4, with expansion of that cohort to treat 3 more patients (total of 6 evaluable patients). One of those next 3 patients developed DLTs consisting of grade- 3 elevations of AST, alkaline phosphatase, and bilirubin levels. The patient had metastatic disease involving his liver. He died after cycle 1 of bortezomib and temozolomide. His death was felt to be due to progressive disease, and his elevated liver function tests were assessed as being possibly related to study treatment.

With 1 out of 6 patients in group B experiencing a DLT on dose level 4, the MTDs had been reached for group B. The MTDs for the combination of bortezomib and temozolomide are $1.3 \mathrm{mg} / \mathrm{m}^{2} /$ dose for bortezomib and $200 \mathrm{mg} / \mathrm{m}^{2} /$ dose for temozolomide in patients not taking concomitant HEIAs. The MTD for group A was not reached. Due to slow enrollment, the arm of the study was closed to accrual when the MTDs for group B were determined.

The median number of treatment cycles was 2 (range 1-6). Table 3 summarizes the grade-2 through grade- 4 toxicities seen in all study patients during cycle 1 of treatment. Although grade-4 neutropenia and thrombocytopenia were DLTs, the most common hematologic toxicity was grade-2 and grade-3 lymphopenia. The most common non-hematologic toxicities were constipation, fatigue, nausea, and anorexia. Disease progression was the most frequent reason for going off study.

\section{Efficacy}

Twelve patients were evaluable for response. Five had stable disease (SD), with a median number of treatment cycles of 4 (range 2-6). One patient with metastatic carcinoid tumor involving his liver (group B) had confirmed SD after 4 cycles of study treatment on dose level 4 . He went on to complete a total of 6 cycles but did not have follow-up scans performed after the 6 th cycle, because he was incarcerated. There were 4 patients who had early deaths due to progressive disease. Two patients with primary brain tumors ( 1 recurrent GBM, 1 recurrent meningioma) had SD after 2 cycles of study treatment.

\section{Pharmacokinetics}

A total of 24 subjects had PK data collected (3 in group A and 21 in group B). On day 2, data were available for 3 and 21 subjects in groups A and B, respectively. On day 9, data were available for 2 and 20 subjects in groups A and B, respectively. Plasma concentration versus time profiles for patients in groups A and B on days 2 and 9 of the first cycle are shown in Fig. 1, and the PK data from days 2 and 9 are summarized in Table 4. As shown in 
the figure, plasma concentrations declined bi-exponentially in both groups, with a rapid initial decline of 100-fold within the first few hours after the dose, followed by a more gradual decay over the next $12-24 \mathrm{~h}$. Bortezomib $\mathrm{CL}_{\mathrm{sys}}$ on day 9 was $51 \%$ (median percent change) of the $\mathrm{CL}_{\text {sys }}$ on day $2(P<0.01$, paired Wilcoxon test). Correspondingly, the normalized AUC on day 9 was 1.9 times (median fold change) the normalized AUC on day $2(P<0.01)$.

During the 12-24-h period following the dose, plasma bortezomib concentrations appeared to fall more rapidly in group A patients compared to those in group B. Moreover, on both days 2 and 9, the mean bortezomib $\mathrm{CL}_{\text {sys }}$ was significantly higher in group A patients compared to those in group B, and similarly, the normalized AUC was lower in group A patients compared to those in group B. In the 3 group A patients on day 2, the median and range for $\mathrm{CL}_{\mathrm{sys}}$ was 94.8 [64.4-115.7] versus 45.8 [16.0-89.6] 1/h in group $\mathrm{B},(P<0.02$, Wilcoxon signed rank test). For the normalized AUC, the median and range, respectively, for the two groups, was 27.0 [26.5-40.4] in group A versus 50.4 [27.4-137.8] in group B, $P$ $<0.02$. On day 9, the median and range $\mathrm{CL}_{\mathrm{sys}}$ was 57.0 [35.9-78.1] versus 20.7 [9.3-59.3] $\mathrm{l} /$ $\mathrm{h}(P<0.04)$, and for normalized AUC 56.2 [40.0-72.4] versus 116.6 [41.7-281.0] $(P<$ $0.04)$. As these results are based on only 3 patients receiving HEIAs, the results should be interpreted with caution.

In terms of concomitant anti-seizure medications, all 3 patients in group A were taking phenytoin, and their serum phenytoin levels were confirmed to be within the therapeutic range at the times of the PK sampling on days 2 and 9 of cycle 1 .

\section{Discussion}

Two other phase I studies of this chemotherapy combination have been reported. Kubicek et al. [39] studied bortezomib and temozolomide in combination with brain radiation for the treatment of CNS malignancies. Su et al. [40] performed a dose escalation study of bortezomib and temozolomide in patients with advanced melanoma. In contrast to our phase 1 trial, these other 2 studies tested bortezomib in combination with a continuous dosing schedule of temozolomide (up to $75 \mathrm{mg} / \mathrm{m}^{2} /$ day for as long as 6 weeks).

Similar to our results, these studies concluded that bortezomib given at $1.3 \mathrm{mg} / \mathrm{m}^{2} /$ dose was well tolerated in combination with temozolomide. Kubicek and colleagues reported that no DLTs were observed in their study patients, but they designed their study without escalation of the dose of bortezomib past $1.3 \mathrm{mg} / \mathrm{m}^{2}$. Different DLTs were seen in our study (constipation, abdominal pain, neutropenia, thrombocytopenia, and hyponatremia) than in the study performed by Su et al. (neurotoxicity, diarrhea, and rash), possibly due to the different dosing schedules of temozolomide (intermittent versus continuous).

Bortezomib is metabolized by multiple cytochrome P450 (CYP) isoforms, with evidence for participation of 5 distinct CYP enzymes based on the results of in vitro enzymology studies [41]. Previous phase I combination studies of bortezomib with multiple different cytotoxic agents have revealed no apparent effects of these concomitantly administered agents on bortezomib pharmacodynamics, although one recent study did demonstrate an effect of the strong CYP3A inhibitor ketoconazole on bortezomib disposition [38]. However, because temozolomide was not expected to affect the systemic elimination of bortezomib and bortezomib was not expected to alter temozolomide PK, assessment of a potential drug-drug interaction between these 2 agents was not an objective of this study.

The PK of bortezomib in the cohort of patients not taking HEIAs determined in the current study are similar to those reported in a recent study by Reece et al. [42] in patients with relapsed multiple myeloma receiving both single and multiple doses of 1.0 and $1.3 \mathrm{mg} / \mathrm{m}^{2}$ of 
bortezomib. Moreover, the MTDs for bortezomib and temozolomide given in combination that were determined in the current study are similar to the tolerable single agent doses. Taken together, our results indicate that there is no PK or PD interaction between these two drugs.

There was a statistically significant repeat-dose effect on bortezomib PK observed in the current study, which is consistent with previous reports of decreased clearance upon multiple bortezomib doses. The mechanism for the effect of multiple doses on bortezomib clearance is unknown.

This phase I study also assessed whether the disposition of bortezomib is affected by drugs that induce hepatic enzymes. Patients with primary or metastatic brain tumors were allowed to participate, and such patients often take medications to control seizures. Certain antiseizure medications, such as phenytoin and carbamazepine, induce the hepatic CYP isoenzyme system. These hepatic enzymes can enhance the clearance of concurrently administered drugs that are eliminated by hepatic oxidative metabolism, resulting in lower blood levels of a drug and decreased efficacy. HEIAs have been shown to reduce the effectiveness of some chemotherapy agents [43], such as paclitaxel [44] and CPT-11 [45].

Temozolomide is not metabolized by the CYP pathway, and thus its serum drug levels are not expected to be affected by the concurrent use of HEIAs. In contrast, the clinical PK of bortezomib in patients taking HEIAs have not yet been described. In mass balance studies of bortezomib conducted in the rat and monkey, radioactivity was eliminated from both renal and hepatic routes [46, 47]. Bortezomib has been shown to be inactivated by both CYP and non-CYP mechanisms [48]. The clinical importance of hepatic metabolism in the elimination of bortezomib has been demonstrated by the finding that the CYP3A4 substrate ketoconazole inhibits bortezomib clearance and results in a mean increase in systemic exposure of $35 \%$ [38].

Phuphanich et al. [49] recently reported the results of a phase I study of single agent bortezomib in 66 adults with recurrent malignant gliomas using separate dose escalations for patients taking or not taking HEIAs. The MTD of bortezomib was $2.5 \mathrm{mg} / \mathrm{m}^{2}$ in the group of patients receiving concurrent HEIAs compared with an MTD of $1.7 \mathrm{mg} / \mathrm{m}^{2}$ in patients not taking HEIAs. Furthermore, the investigators determined that the bortezomib dose at which maximal inhibition of whole blood 20S proteasome activity occurred was higher in patients receiving HEIAs. The authors concluded that concurrently administered HEIAs enhanced the clearance of bortezomib, resulting in lower than expected plasma levels and anti-20S proteasome activity of bortezomib in these patients. PK data were not available to confirm their conclusion because a sensitive analytical method to measure bortezomib in plasma was not available. However, the PK results from our study indicate that the rate of systemic elimination of bortezomib is indeed increased by concurrently administered HEIAs. Although the sample size of the study is small, a significant twofold increase in the mean clearance of bortezomib was seen in patients taking HEIAs compared to those not taking HEIAs. Therefore, the PK findings of this study are consistent with the previous clinical findings of Phuphanich et al. [49].

We determined that the recommended doses for testing this combination in phase 2 studies of patients not taking HEIAs is $1.3 \mathrm{mg} / \mathrm{m}^{2}$ on days $2,5,9$ and 12 for bortezomib and 200 $\mathrm{mg} / \mathrm{m}^{2}$ on days $1-5$ for temozolomide, repeated every 28 days. Due to slow accrual to group A, enrollment was stopped when dose escalation was completed for group B. One reason for the poor accrual to group A is that during the time this trial was open for enrollment, results of a landmark study [25] were published showing a survival benefit for glioblastoma patients when temozolomide was added to radiation in the upfront setting. As a result, there 
were fewer than anticipated brain tumor patients available to participate in this study, since typically they had already been treated with the same dose schedule of temozolomide as part of their first-line therapy. A second possible reason for the poor accrual to group A is the increasing use of second generation anticonvulsants such as levetiracetam, lamotrigine, and zonisamide, which do not induce hepatic enzymes.

Although our study did not enroll a sufficient number of patients in group A to definitively determine the MTDs of bortezomib and temozolomide in patients taking HEIAs, further PK studies in this subgroup of patients are warranted given the preliminary evidence of an induction bortezomib elimination by HEIAs in the 3 patients presented. Temozolomide is used mainly for the treatment of patients with gliomas and advanced melanoma. Melanoma has a propensity to metastasize to the brain. Since patients with CNS malignancies frequently require anti-seizure medication, concurrent use of HEIAs should be prohibited in future efficacy studies of the regimen until the MTDs of this combination can be established in this patient population.

\section{Acknowledgments}

We would like to thank Ms. Brenda Williams, the Phase I research nurse for this study. Grant Support: NIH training grant (K12 CA01727; J. Portnow), and a Cancer Center Support Grant (P30 CA33572).

\section{References}

1. Adams J. The proteasome: structure, function, and role in the cell. Cancer Treat Rev. 2003; 29(Suppl 1):3-9. [PubMed: 12738238]

2. Cusack JC. Rationale for the treatment of solid tumors with the proteasome inhibitor bortezomib. Cancer Treat Rev. 2003; 29(Suppl 1):21-31. [PubMed: 12738240]

3. Adams J, Palombella VJ, Sausville EA, et al. Proteasome inhibitors: a novel class of potent and effective antitumor agents. Cancer Res. 1999; 59:2615-2622. [PubMed: 10363983]

4. Frankel A, Man S, Elliott P, et al. Lack of multicellular drug resistance observed in human ovarian and prostate carcinoma treated with the proteasome inhibitor PS-341. Clin Cancer Res. 2000; 6:3719-3728. [PubMed: 10999766]

5. Williams SA, Papandreou C, McConkey D. Preclinical effects of proteasome inhibitor PS-341 in combination chemotherapy for prostate cancer. Proc Am Soc Clin Oncol. 2001; 20:169b.

6. Shah SA, Potter MW, McDade TP, et al. 26S proteasome inhibition induces apoptosis and limits growth of human pancreatic cancer. J Cell Biochem. 2001; 82:110-122. [PubMed: 11400168]

7. Bold RJ, Virudachalam S, McConkey DJ. Chemosensitization of pancreatic cancer by inhibition of the 26S proteasome. J Surg Res. 2001; 100:11-17. [PubMed: 11516199]

8. Nawrocki ST, McConkey DJ. The proteasome inhibitor, PS-341, inhibits the growth of pancreatic cancer cells in vitro and in vivo. Proc Am Assoc Cancer Res. 2002; 43:159.

9. Sclabas GM, Dong Qg, Fujioka S, et al. Drug-elicited apoptosis in pancreatic tumor cells: the role of different complexes between IkB and NF-kB. Proc Am Assoc Cancer Res. 2002; 43:882.

10. Ling YH, Jiang JD, Liebes L, et al. Molecular mechanisms of proteasome inhibitor PS-341induced G2/M phase arrest and apoptosis in human non-small cell lung cancer $\mathrm{H} 460$ cells. Proc Am Assoc Cancer Res. 2002; 43:665.

11. Gumerlock PH, Moisan LP, Lau AH, et al. Docetaxel followed by PS-341 results in phosphorylation and stabilization of p27 and increases response in non-small cell lung carcinoma (NSCLC). Clin Cancer Res. 2001; 7:157.

12. Sunwoo JB, Chen Z, Dong G, et al. Novel proteasome inhibitor PS-341 inhibits activation of nuclear factor $\mathrm{kB}$, cell survival, tumor growth, and angiogenesis in squamous cell carcinoma. Clin Cancer Res. 2001; 7:1419-1428. [PubMed: 11350913]

13. Chen Z, Malhotra PS, Gill R, et al. Identification of sensitive and resistant head and neck squamous cell carcimoma lines to PS-341, a novel anti-cancer agent inhibiting proteasome dependent activation of NF-kB. Proc Am Assoc Cancer Res. 2002; 43:158. 
14. Cusack JC, Liu R, Houston M, et al. Enhanced chemosensitivity to CPT-11 with proteasome inhibitor PS-341: implications for systemic nuclear factor-kB inhibition. Cancer Res. 2001; 61:3535-3540. [PubMed: 11325813]

15. Teicher BA, Ara G, Herbst R, et al. The proteasome inhibitor PS-341 in cancer therapy. Clin Cancer Res. 1999; 5:2638-2645. [PubMed: 10499643]

16. Thornton, JD.; Liu, R.; Orlowski, RZ., et al. Doxorubicin-induced NF-kappaB activation in breast cancer is overcome by proteasome inhibition, resulting in enhanced tumoricidal response to treatment. Presented at the 87th annual clinical congress of the American college of surgeons; New Orleans. October 7-12; 2001.

17. Kamat AM, Karashima T, Dinney CP, et al. The proteasome inhibitor PS-341 sensitizes drugrefractory human transitional cell tumors to gemcitabine. Proc Am Assoc Cancer Res. 2002; 43:157.

18. Pink M, Pien CS, Worland P, et al. PS-341 enhances chemo-therapeutic effect in human xenograft models. Proc Am Assoc Cancer Res. 2002; 43:158.

19. Ryan DP, Appleman LJ, Lynch T, et al. Phase I clinical trial of bortezomib in combination with gemcitabine in patients with advanced solid tumors. Cancer. 2006; 15:2482-2489. [PubMed: 17036355]

20. Ryan DP, O’Neill BH, Supko JG, et al. A phase I study of bortezomib plus irinotecan in patients with advanced solid tumors. Cancer. 2006; 107:2688-2697. [PubMed: 17075878]

21. Messersmith WA, Baker SD, Lassiter L, et al. Phase I trial of bortezomib in combination with Docetaxel in patients with advanced solid tumors. Clin Cancer Res. 2006; 12:1270-1275. [PubMed: 16489083]

22. Cohen SJ, Engstrom PF, Lewis NL, et al. Phase I study of capecitabine and oxaliplatin in combination with the proteasome inhibitor bortezomib in patients with advanced solid tumors. Am J Clin Oncol. 2008; 31:1-5. [PubMed: 18376220]

23. Ma C, Mandrekar SJ, Alberts SR, et al. A phase I and pharmacologic study of sequences of the proteasome inhibitor, bortezomib (PS-341, Velcade), in combination with paclitaxel and carboplatin in patients with advanced malignancies. Cancer Chemother Pharmacol. 2007; 59:207215. [PubMed: 16763792]

24. Dees EC, O'Neil BH, Lindley CM, et al. A phase I and pharmacologic study of the combination of bortezomib and pegylated liposomal doxorubicin in patients with refractory solid tumors. Cancer Chemother Pharmacol. 2008; 63:99-107. [PubMed: 18327587]

25. Stupp R, Mason WP, van den Bent MJ, et al. Radiotherapy plus comcomitant and adjuvant temozolomide for glioblastoma. New Eng J Med. 2005; 352:987-996. [PubMed: 15758009]

26. Quirt I, Verma S, Petrella T, et al. Temozolomide for the treatment of metastatic melanoma: a systemic review. Oncologist. 2007; 12:1114-1123. [PubMed: 17914081]

27. Gunther W, Pawlak E, Damasceno R, et al. Temozolomide induces apoptosis and senescence in glioma cells cultured as multicellular spheroids. Br J Cancer. 2003; 88:463-469. [PubMed: 12569392]

28. Amiri KI, Horton LW, LaFleur BJ, et al. Augmenting chemosensitivity of malignant melanoma tumors via proteasome inhibition: implications for bortezomib (VELCADE, PS-341) as a therapeutic agent for malignant melanoma. Cancer Res. 2004; 64:4912-4918. [PubMed: 15256463]

29. Abrey LE, Olson JD, Raizer JJ, et al. A phase II trial of temozolomide for patients with recurrent or progressive brain metastases. J Neurooncol. 2001; 53:259-265. [PubMed: 11718258]

30. Christodoulou C, Bafaloukos D, Kosmidis P, et al. Phase II study of temozolomide in heavily pretreated cancer patients with brain metastases. Ann Oncol. 2001; 12:249-254. [PubMed: 11300333]

31. Antonadou D, Paraskevaidis M, Sarris G, et al. Phase II randomized trial of temozolomide and concurrent radiotherapy in patients with brain metastases. J Clin Oncol. 2002; 20:3644-3650. [PubMed: 12202665]

32. Aghajanian C, Soignet S, Dizon DS, et al. A phase I trial of the novel proteasome inhibitor PS-341 in advanced solid tumor malignancies. Clin Cancer Res. 2002; 8:2505-2511. [PubMed: 12171876] 
33. Papandreou CN, Daliani DD, Nix D, et al. Phase I trial of the proteasome inhibitor bortezomib in patients with advanced solid tumors with observations in androgen-independent prostate cancer. $\mathbf{J}$ Clin Oncology. 2004; 22:2108-2121.

34. Newlands ES, Blackledge GR, Slack JA, et al. Phase I trial of temozolomide (CCRG 81045: M\&B 39831: NSC 3628560. Br J Cancer. 1992; 65:287-291. [PubMed: 1739631]

35. Brada M, Judson I, Beale P, et al. Phase I dose-escalation and pharmacokinetic study of temzolomide (SCH 52365) for refractory or relapsing malignancies. Br J Cancer. 1999; 81:10221030. [PubMed: 10576660]

36. Therasse P, Arbuck SG, Eisenhauer EA, et al. New guidelines to evaluate the response to treatment in solid tumors. J Natl Cancer Inst. 2000; 92:205-216. [PubMed: 10655437]

37. Macdonald DR, Cascino TL, Schold SC Jr, Cairncross JG. Response criteria for phase II studies of supratentorial malignant glioma. J Clin Oncol. 1990; 8:1277-1280. [PubMed: 2358840]

38. Venkatakrishnan K, Rader M, Ramanathan RK, et al. Effect of the CYP3A inhibitor ketoconazole on the pharmacokinetics and pharmacodynamics of bortezomib in patients with advanced solid tumors: a prospective, multicenter, open-label, randomized, two-way crossover drug-drug interaction study. Clin Ther. 2009; 31:2444-2458. [PubMed: 20110052]

39. Kubicek GJ, Werner-Wasik M, Machtay M, et al. Phase I trial using proteasome inhibitor bortezomib and concurrent temozolomide and radiotherapy for central nervous system malignancies. Int J Radia Oncol Biol Phys. 2009; 74:433-439.

40. Su Y, Amiri KI, Horton LW, et al. A phase I trial of bortezomib with temozolomide in patients with advanced melanoma: toxicities, antitumor effects, and modulation of therapeutic targets. Clin Cancer Res. 2010; 16:348-357. [PubMed: 20028756]

41. Uttamsingh V, Lu C, Miwa G, Gan LS. Relative contributions of the five major human cytochromes P450, 1A2, 2C9, 2C19, 2D6, and 3A4, to the hepatic metabolism of the proteasome inhibitor bortezomib. Drug Metab Dispos. 2005; 33:1723-1728. [PubMed: 16103134]

42. Reece DE, Sullivan D, Lonial S, et al. Pharmacokinetic and pharmacodynamic study of two doses of bortezomib in patients with relapsed multiple myeloma. Cancer Chemother Pharmacol. 2011; 67:57-67. [PubMed: 20306195]

43. Vecht CJ, Wagner GL, Wilms EB. Interactions between antiepileptic and chemotherapeutic drugs. Lancet Neurol. 2003; 2:404-409. [PubMed: 12849118]

44. Rowinsky EK. Paclitaxel pharmacology and other tumor types. Semin Oncol. 1997; 24:S19-1S19-12. [PubMed: 9427257]

45. Gilbert MR, Supko JG, Batchelor T, et al. Phase I clinical and pharmacokinetic study of irinotecan in adults with recurrent malignant glioma. Clin Cancer Res. 2003; 9:2940-2949. [PubMed: 12912940]

46. Nix D, Press RJ, Wehrman TG. Tissue distribution, mass balance, and biliary excretion of bortezomib (VELCADE ${ }^{\mathrm{TM}}$ ) in male rats. Drug Metab Rev. 2003; 35(2):224.

47. Nix D, Wherman TG, Press RJ. Tissue distribution and mass balance of bortezomib $\left(\right.$ VELCADE $^{\mathrm{TM}}$ ) in non-human primates. AAPS PharmSci. 2003; 5:4. (Abstract M1336).

48. Investigator Brochure for PS-341. 13. Millennium pharmaceuticals, Inc; 2010.

49. Phuphanich S, Supko JG, Carson KA, et al. Phase 1 clinical trial of bortezomib in adults with recurrent malignant glioma. J Neurooncol. 2010; 100:95-103. [PubMed: 20213332] 

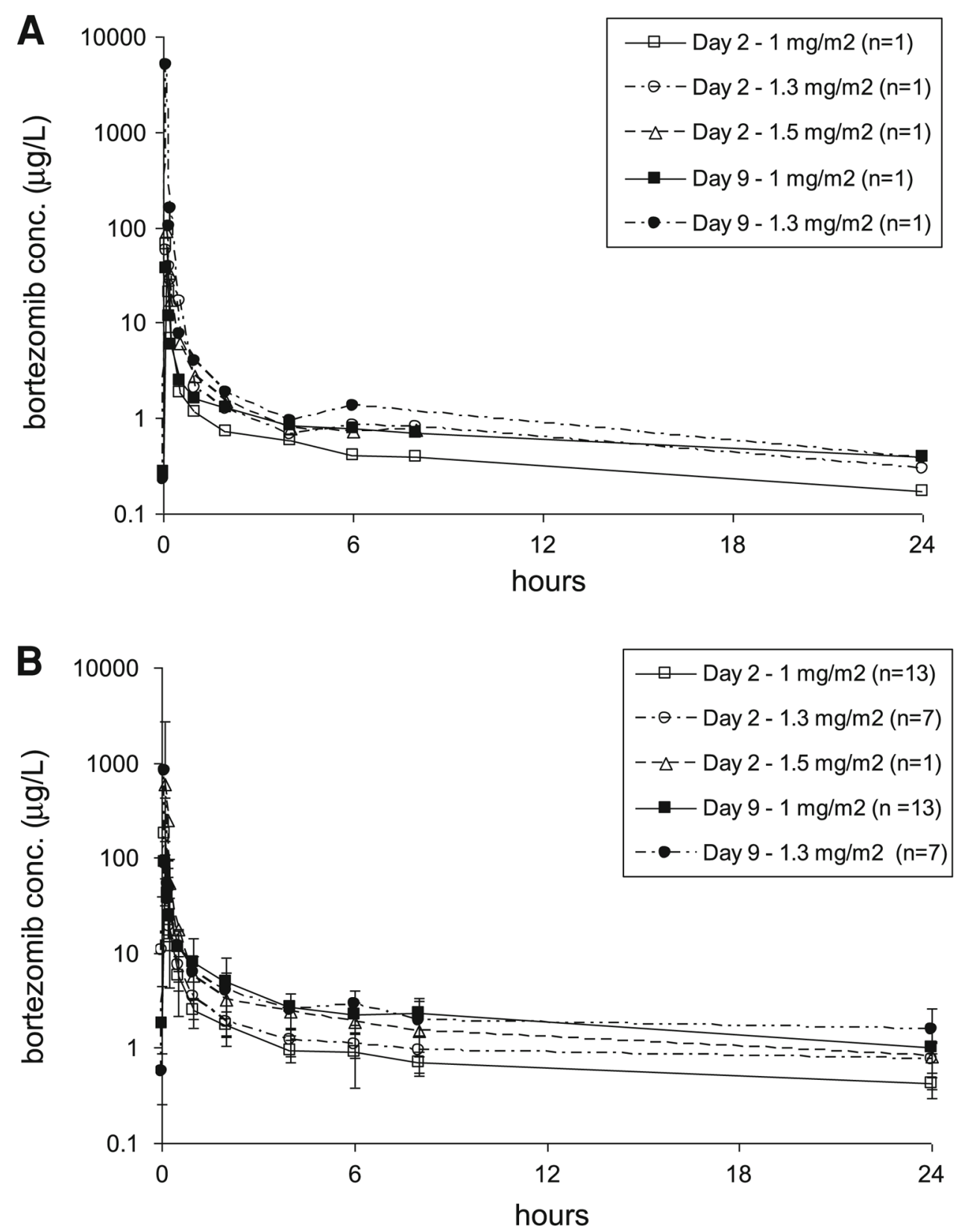

Fig. 1.

Plasma bortezomib concentration versus time profiles for study patients taking (group A) and not taking (group B) concomitant HEIAs. Data points and error bars in panel B represent the means and standard deviations 


\section{Table 1}

Patient characteristics $(n=25)$

\begin{tabular}{|c|c|}
\hline Characteristics & No. of patients \\
\hline Age (years) [median (range)] & $52(23-81)$ \\
\hline \multicolumn{2}{|l|}{ Gender } \\
\hline Female & 9 \\
\hline Male & 16 \\
\hline \multicolumn{2}{|l|}{ Race } \\
\hline African-American & 3 \\
\hline Asian & 5 \\
\hline Caucasian & 17 \\
\hline \multicolumn{2}{|l|}{ Primary tumor } \\
\hline Colon & 5 \\
\hline Lung & 5 \\
\hline Primary brain tumor & 4 \\
\hline Breast & 2 \\
\hline Prostate & 2 \\
\hline Adenoid cystic carcinoma & 2 \\
\hline Carcinoid tumor & 2 \\
\hline Other ${ }^{a}$ & 3 \\
\hline \multicolumn{2}{|l|}{ Karnofsky performance status } \\
\hline Median & 80 \\
\hline Range & $(60-100)$ \\
\hline Median no. of prior chemotherapy regimens (range) & $3(0-6)$ \\
\hline \multicolumn{2}{|l|}{ Group } \\
\hline A & 3 \\
\hline $\mathrm{B}$ & 22 \\
\hline
\end{tabular}

${ }^{a}$ Appendiceal carcinoma $(n=1)$, gastric carcinoma $(n=1)$, and adenocarcinoma of unknown primary $(n=1)$ 


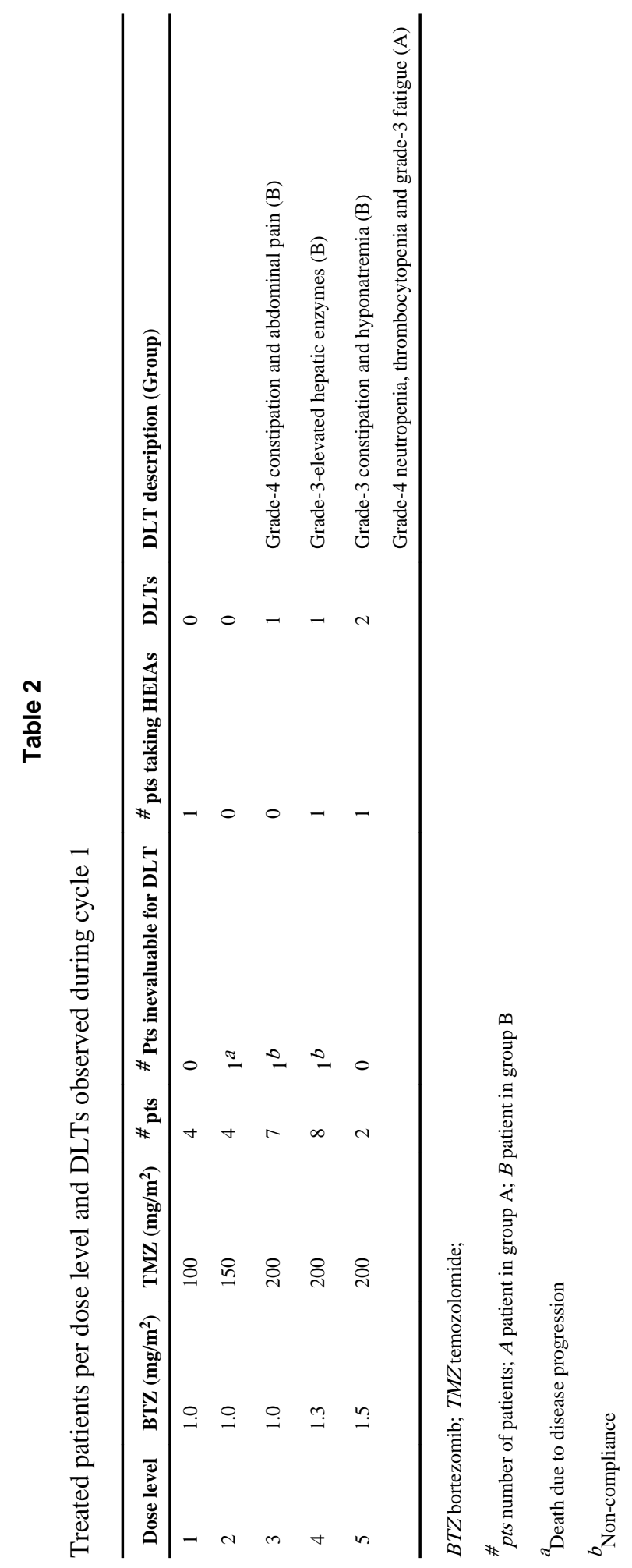

Cancer Chemother Pharmacol. Author manuscript; available in PMC 2013 February 01. 
Table 3

Summary of adverse events ( zgrade 2) during cycle 1 in all patients

\begin{tabular}{|c|c|c|c|}
\hline Parameter & Grade 2 & Grade 3 & Grade 4 \\
\hline \multicolumn{4}{|l|}{ Hematologic } \\
\hline Neutropenia & & & 1 \\
\hline Lymphopenia & 8 & 10 & \\
\hline Anemia & 3 & 2 & \\
\hline Thrombocytopenia & 2 & 4 & 1 \\
\hline \multicolumn{4}{|l|}{ Non-hematologic } \\
\hline Alkaline phosphatase & 1 & 1 & \\
\hline Alkalosis (metabolic or respiratory) & 1 & & \\
\hline ALT/SGPT & 1 & & \\
\hline Anorexia & 4 & & \\
\hline AST/SGOT & & 1 & \\
\hline Bicarbonate, serum-low & 1 & & \\
\hline Bilirubin & & 1 & \\
\hline Constipation & 3 & 1 & 1 \\
\hline Creatinine & 1 & & \\
\hline Dehydration & 1 & & \\
\hline Dermatology/skin & 1 & & \\
\hline Diarrhea & 2 & & \\
\hline Distension/abdominal bloating & 1 & & \\
\hline Dizziness & 1 & & \\
\hline Edema: limb & 2 & & \\
\hline Fatigue & 11 & 1 & \\
\hline Hyperglycemia & 2 & & \\
\hline Hypoalbuminemia & 3 & & \\
\hline Hypocalcemia & 2 & & \\
\hline Hyponatremia & & 1 & \\
\hline Hypothyroidism & 1 & & \\
\hline Infection without neutropenia & 1 & & \\
\hline Insomnia & 1 & & \\
\hline Mood alteration & 1 & & \\
\hline Mucositis/stomatitis & 1 & & \\
\hline Muscle weakness & & 2 & \\
\hline Nausea & 9 & & \\
\hline Neuropathy: motor & 2 & & \\
\hline Pain & 2 & & 1 \\
\hline Pericarditis & 1 & & \\
\hline Pruritis & 1 & & \\
\hline Vomiting & 1 & & \\
\hline Weight loss & 1 & & \\
\hline
\end{tabular}

Cancer Chemother Pharmacol. Author manuscript; available in PMC 2013 February 01. 


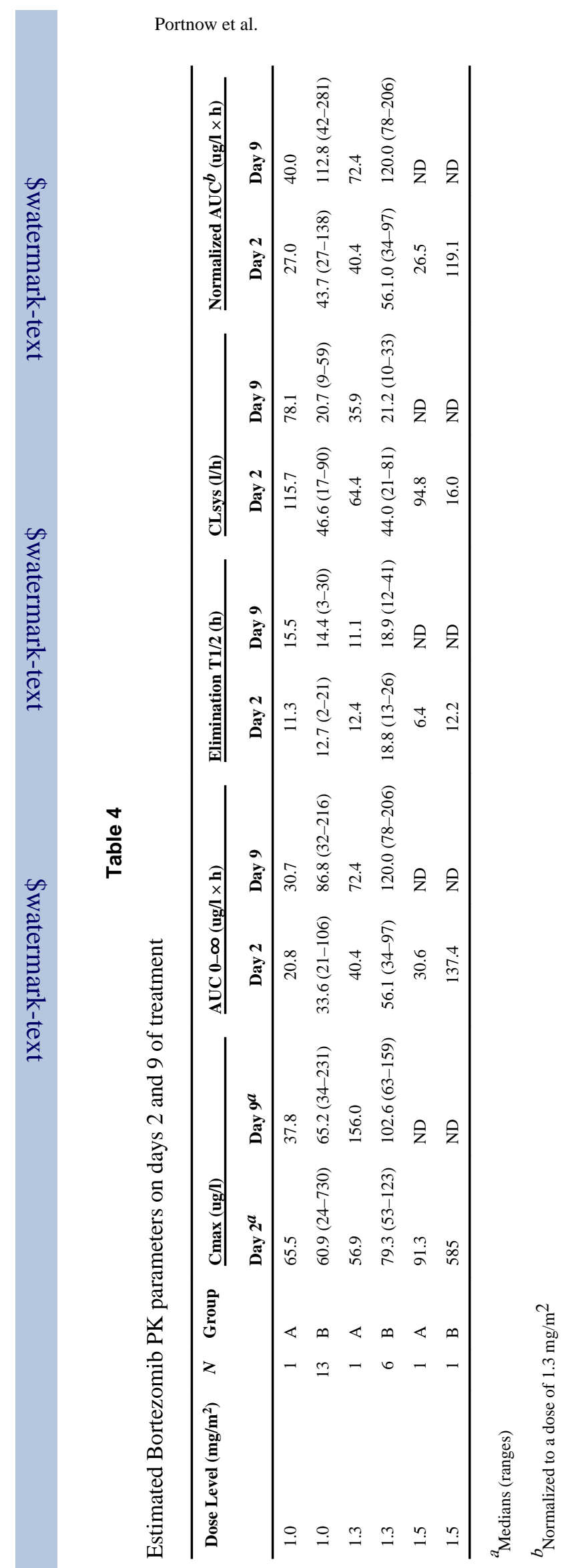

Page 16 